Supporting Information for

\title{
Defining nutrient co-location typologies for human-derived supply and crop demand to advance resource recovery
}

\author{
Desarae Echevarria, John T. Trimmer, Roland D. Cusick, Jeremy S. Guest
}

\begin{abstract}
Table of Contents (10 total pages)

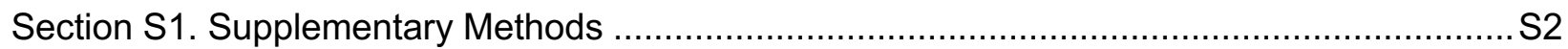

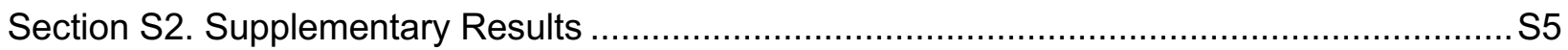

Section S3. Nutrient Saturation Profile Plots of All Countries ................................................ 10

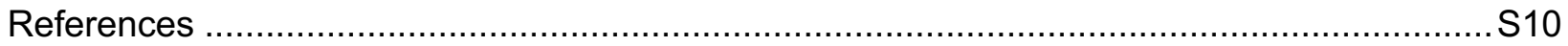

\section{List of Figures}

Figure S1. Validation of the SPAM model by comparing the sum of seven crop areas in 179 countries to FAO reports

Figure S2. Nutrient contribution stratification among grid cell classifications for nitrogen,

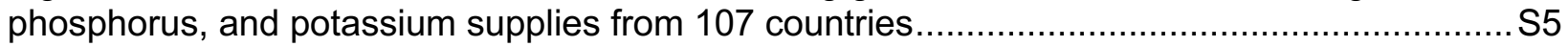

Figure S3. Nitrogen principal component analysis (PCA) using HDI grouping .......................... 6

Figure S4. Phosphorus principal component analysis (PCA) using HDI grouping ….................S7

Figure S5. Potassium principal component analysis (PCA) using HDI grouping........................ 8

\section{List of Tables}

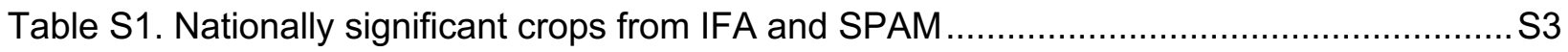

Table S2. FAO crops used to calculate nationally significant crop area .................................... 4

Table S3. Nationally significant crop fractions for 107 countries .......................................... 4

Table S4. Nitrogen nutrient stratification among grid cell classifications and fitting parameters for N-Nutrient Saturation Profiles of 107 countries ………..................................................... S5

Table S5. Phosphorus nutrient stratification among grid cell classifications and fitting parameters for P-Nutrient Saturation Profiles of 107 countries

Table S6. Potassium nutrient stratification among grid cell classifications and fitting parameters for K-Nutrient Saturation Profiles of 107 countries S5

Table S7. Nitrogen, phosphorus, and potassium PCA results showing clusters and country characteristics 


\section{Section S1. Supplementary Methods}

Principal component analysis (PCA) transforms a dataset of potentially interrelated variables into a set of linearly uncorrelated principal components. PCA was conducted on a combination of (i) data-derived parameters, $<S$ and $S$, and (ii) generalized logistic function fitting parameters $(X, M$, and log-transformed $B$ and $\mu$ ) for 92 countries (15 datasets were removed as outliers). Of the 15 removed countries, we removed 12 with fewer than 20 grid cells containing supply or demand data (as these offered a very small number of data points, making reliable curve fitting difficult). We also removed 3 countries (Iceland, Cyprus, Brunei Darussalam) because curve fitting resulted in extreme values for certain parameters (e.g., $\mu$ values $>2$ orders of magnitude larger than those of other countries). These values overshadowed all other distinctions and trends that the PCA would have helped us to see. We describe one example (Iceland) in detail in the main text.

Each principal component represents a linear combination of the original set of variables, which then provides the position of each country within the transformed dataset. To remove differences in scale prior to PCA, each variable was transformed such that its mean equaled zero and standard deviation equaled one. ${ }^{1,2}$ To identify clustering in the PCA beyond the input parameters, we plotted the first two principal components (representing approximately two-thirds of the total variance in our data) and investigated trends related to several country groupings, including the Human Development Index (HDI, with HDI 1 being the highest and HDI 4 the lowest based on UN Development Programme categories), income group (defined by the World Bank), urban population as a percent of total population, percent of the workforce employed in agriculture, total fertilizer consumption in kilograms of nutrients $(\mathrm{N}+\mathrm{P}+\mathrm{K})$ per hectare (all grouped based on the $25^{\text {th }}, 50^{\text {th }}$, and $75^{\text {th }}$ percentile values from the full distribution across all countries), and geographic region. Clusters were most apparent with the HDI country characteristic grouping, and so we focused primarily on the relationship between nutrient saturation profiles and $\mathrm{HDI}$ tier for $\mathrm{N}$ (Figure S3; Table S7). The same analysis was conducted for P and K (Figures S4 and S5; Table S7), which followed similar trends. Of the 92 countries (and territories) used to complete all PCAs, 90 had HDI measurements (Puerto Rico and Somalia did not). It is worth noting that an ANOVA test showed there is not a significant difference $(p=0.535)$ among the average number of grid cells across the three clusters.

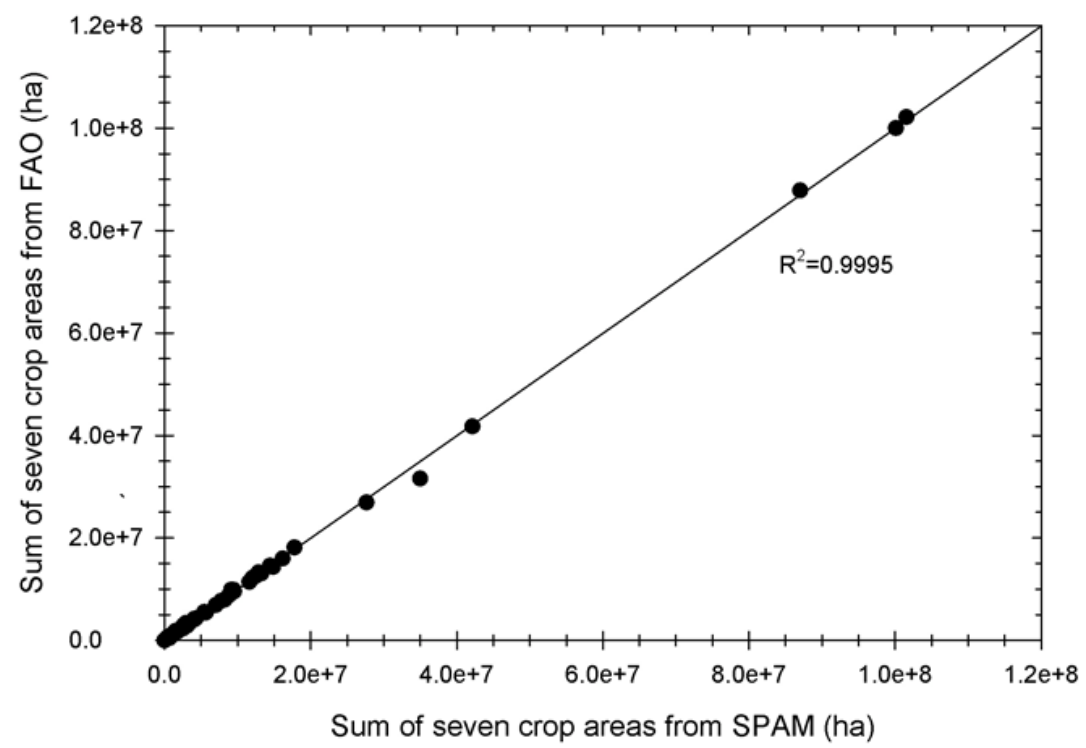

Figure S1. Validation of the SPAM model by comparing the sum of seven crop areas in 179 countries to FAO reports. 
Table S1. Nationally significant crops from IFA and SPAM.

\begin{tabular}{|l|l|}
\hline $\begin{array}{c}\text { Nationally significant } \\
\text { Crop name from IFA's } \\
\text { World Fertilizer Manual }\end{array}$ & $\begin{array}{c}\text { Crop from SPAM included in } \\
\text { spatial analysis }\end{array}$ \\
\hline coffee, green & $\begin{array}{l}\text { arabica coffee, robusta } \\
\text { coffee }\end{array}$ \\
\hline bananas & banana \\
\hline barley & barley \\
\hline beans, dry & bean \\
\hline cassava & cassava \\
\hline coconut & coconut \\
\hline cocoa, beans & cocoa \\
\hline cotton & cotton \\
\hline cow peas, dry & cowpea \\
\hline groundnut & groundnut \\
\hline lentils & lentil \\
\hline maize & maize \\
\hline cereal (other) & other cereals \\
\hline oil palm & oilpalm \\
\hline pulses (other) & other pulses \\
\hline roots (other) & other roots \\
\hline plantains & plantain \\
\hline millet & pearl millet, small millet \\
\hline potato & potato \\
\hline rapeseed & rapeseed \\
\hline rice & rice \\
\hline sorghum & sorghum \\
\hline soybean & soybean \\
\hline sugar beet & sugarbeet \\
\hline sugar cane & sugarcane \\
\hline sunflower & sunflower \\
\hline sweet potatoes & sweet potato \\
\hline tea & tea \\
\hline fruit, tropical (other) & \\
\hline vegetables (other) & vegetables \\
\hline wheat & yams \\
\hline yams & \\
\hline
\end{tabular}


Table S2. FAO crops used to calculate nationally significant crop area.

\begin{tabular}{|c|c|c|}
\hline Apples & Fonio & Plantains and others \\
\hline Artichokes & Fruit, citrus nes & Plums and sloes \\
\hline Asparagus & Fruit, fresh nes & Potatoes \\
\hline Bambara beans & Fruit, pome nes & Pulses, nes \\
\hline Bananas & Fruit, stone nes & Pumpkins, squash and gourds \\
\hline Barley & Fruit, tropical fresh nes & Quinces \\
\hline Beans, dry & Garlic & Quinoa \\
\hline Beans, green & Gooseberries & Rapeseed \\
\hline Berries nes & Grain, mixed & Raspberries \\
\hline Blueberries & Grapefruit (inc. pomelos) & Rice, paddy \\
\hline $\begin{array}{l}\text { Broad beans, horse beans, } \\
\text { dry }\end{array}$ & Grapes & Roots and tubers, nes \\
\hline Buckwheat & Groundnuts, with shell & Rye \\
\hline $\begin{array}{l}\text { Cabbages and other } \\
\text { brassicas }\end{array}$ & Kiwi fruit & Seed cotton \\
\hline Canary seed & $\begin{array}{l}\text { Leeks, other alliaceous } \\
\text { vegetables }\end{array}$ & Sorghum \\
\hline Carobs & Lemons and limes & Soybeans \\
\hline Carrots and turnips & Lentils & Spinach \\
\hline Cassava & Lettuce and chicory & Strawberries \\
\hline Cassava leaves & Lupins & String beans \\
\hline Cauliflowers and broccoli & Maize & Sugar beet \\
\hline Cereals, nes & Maize, green & Sugar cane \\
\hline Cherries & Millet & Sunflower seed \\
\hline Cherries, sour & Mushrooms and truffles & Sweet potatoes \\
\hline Chicory roots & Oats & $\begin{array}{l}\text { Tangerines, mandarins, } \\
\text { clementines, satsumas }\end{array}$ \\
\hline Chillies and peppers, green & Oil palm fruit & Taro (cocoyam) \\
\hline Cocoa, beans & Okra & Tea \\
\hline Coconuts & Onions, dry & Tomatoes \\
\hline Coffee, green & Onions, shallots, green & Triticale \\
\hline Cow peas, dry & Oranges & Vegetables, fresh nes \\
\hline Cranberries & Peaches and nectarines & Vegetables, leguminous nes \\
\hline Cucumbers and gherkins & Pears & Vetches \\
\hline Currants & Peas, dry & Wheat \\
\hline Eggplants (aubergines) & Peas, green & Yams \\
\hline & & Yautia (cocoyam) \\
\hline
\end{tabular}

Table S3. Nationally significant crop fractions for 107 countries.

(Table S3 is included as an excel spreadsheet:

Echevarria_et_al_-_Table_S3_Nationally_Significant_Crop_Fractions.xIsx) 


\section{Section S2. Supplementary Results}

Table S4. Nitrogen nutrient stratification among grid cell classifications and fitting parameters for $\mathrm{N}$-nutrient saturation profiles of 107 countries.

(Table 54 is included as an excel spreadsheet:

Echevarria_et_al_-_Table_S4_Nitrogen_Fitting_Parameters_107_countries.xlsx)

Table S5. Phosphorus nutrient stratification among grid cell classifications and fitting parameters for Pnutrient saturation profiles of 107 countries.

(Table S5 is included as an excel spreadsheet:

Echevarria_et_al_-_Table_S5_Phosphorus_Fitting_Parameters_107_countries.xlsx)

Table S6. Potassium nutrient stratification among grid cell classifications and fitting parameters for Knutrient saturation profiles of 107 countries.

(Table S6 is included as an excel spreadsheet:

Echevarria_et_al_-_Table_S6_Potassium_Fitting_Parameters_107_countries.xlsx)

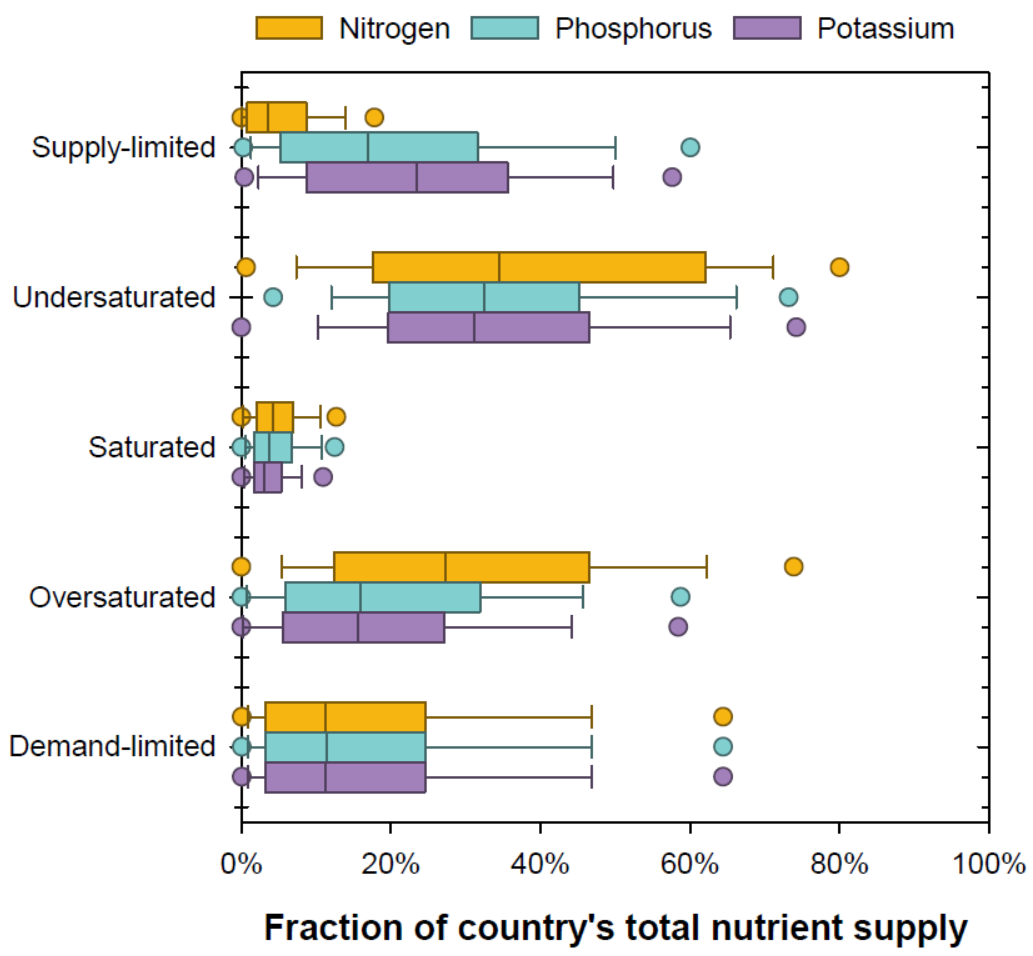

Figure S2. Nutrient contribution stratification among grid cell classifications for nitrogen, phosphorus, and potassium supplies from 107 countries. The $5^{\text {th }}$ and $95^{\text {th }}$ percentiles are shown with points, the 10 th (end of lower whisker), 25th (lower end of box), 50th (median line inside the box), 75th (upper end of box), 90th (end of upper whisker) percentiles are also shown. 

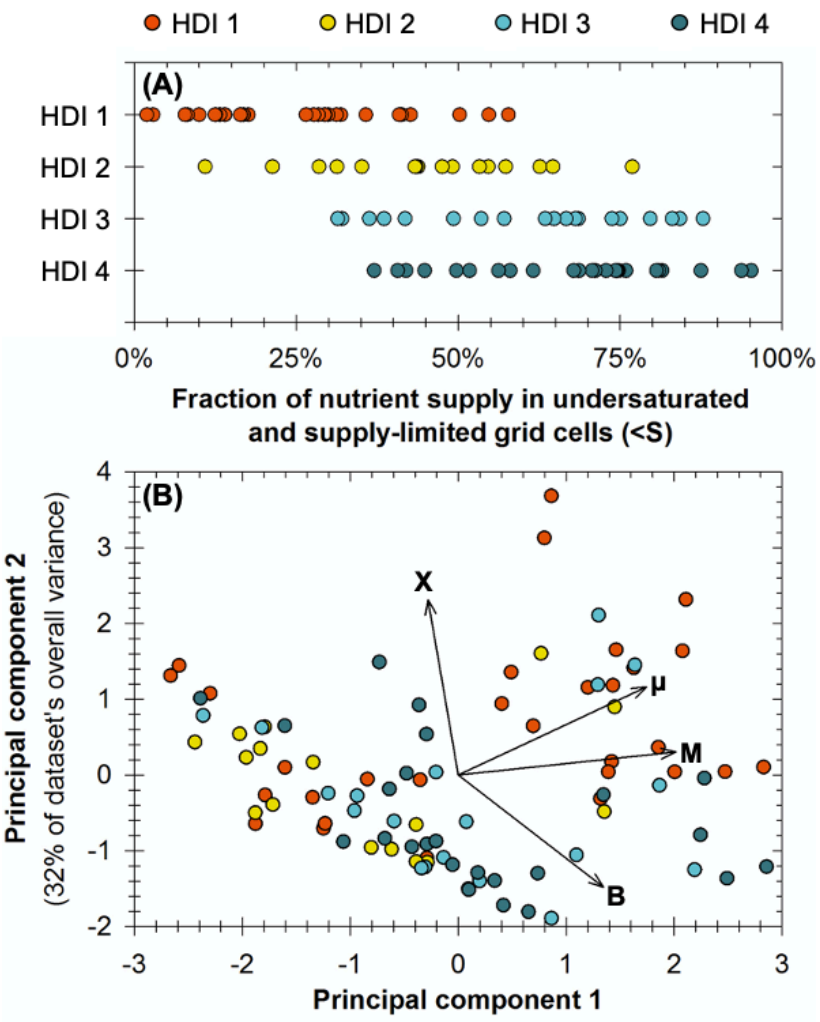

(representing $52 \%$ of the dataset's overall variance)

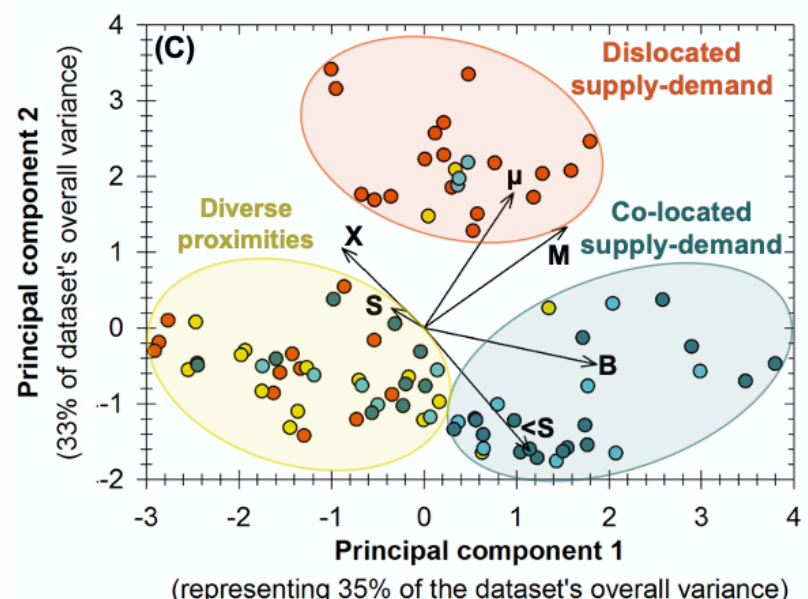

Figure S3. Nitrogen principal component analysis (PCA) using HDI grouping. PCA using $N$ datasets and $\mathrm{HDI}$ grouping to identify clusters using (A) data-derived parameters, $<S$ and $S$, (B) logistic function fitting parameters (after log transforming $B$ and $\mu$ parameters), and $(C)$ a combination of both. Classification of countries into each typology (co-located, dislocated, and diverse proximities) for nitrogen was based on Figure (C). Puerto Rico and Somalia were excluded because they did not have a HDI measurement; thus, only 90 countries are shown. 


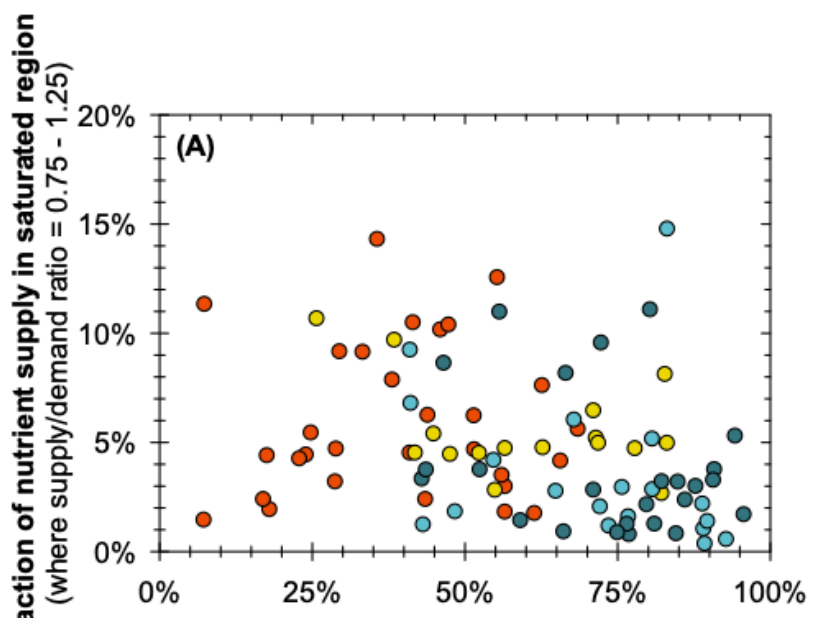

Fraction of nutrient supply below saturated region (where supply/demand ratio $<0.75$ )

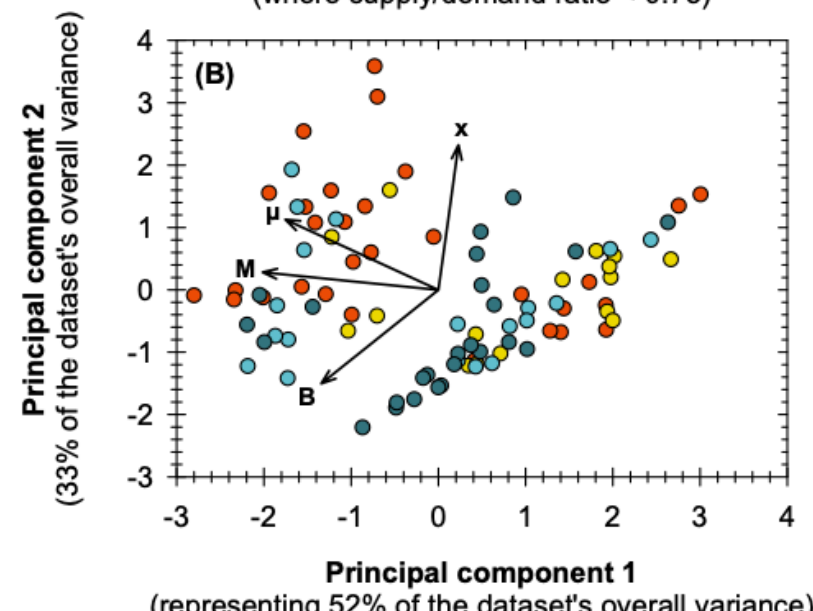

(representing $52 \%$ of the dataset's overall variance)

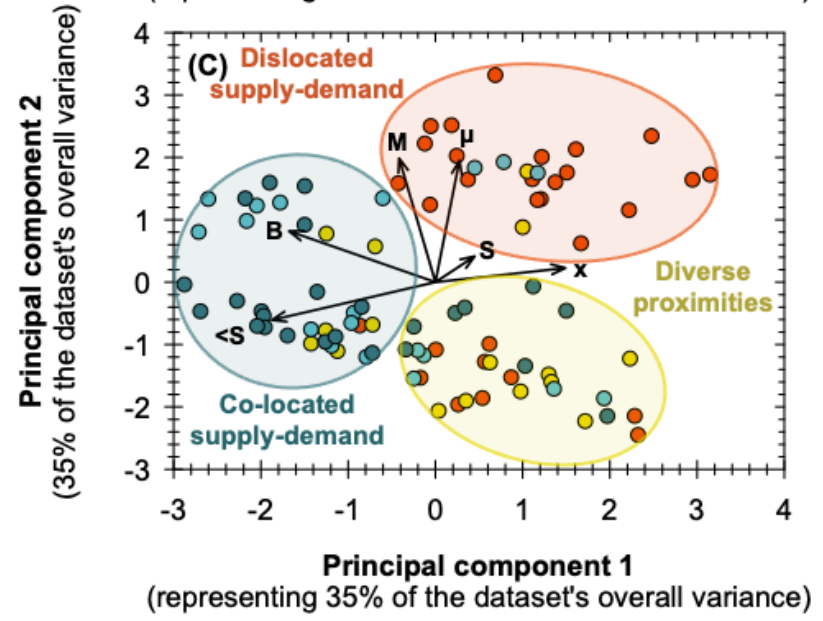

- HDI 1 ○ HDI $2 \circ$ HDI 3 ค HDI 4

Figure S4. Phosphorus principal component analysis (PCA) using HDI grouping. PCA using $P$ datasets and $\mathrm{HDI}$ grouping to identify clusters using $(A)$ data-derived parameters, $<S$ and $S$, (B) logistic function fitting parameters (after log transforming $B$ and $\mu$ parameters), and $(C)$ a combination of both. Classification of countries into each typology (co-located, dislocated, and diverse proximities) for phosphorus was based on Figure $(\mathrm{C})$. Puerto Rico and Somalia were excluded because they did not have a HDI measurement; thus, only 90 countries are shown. 


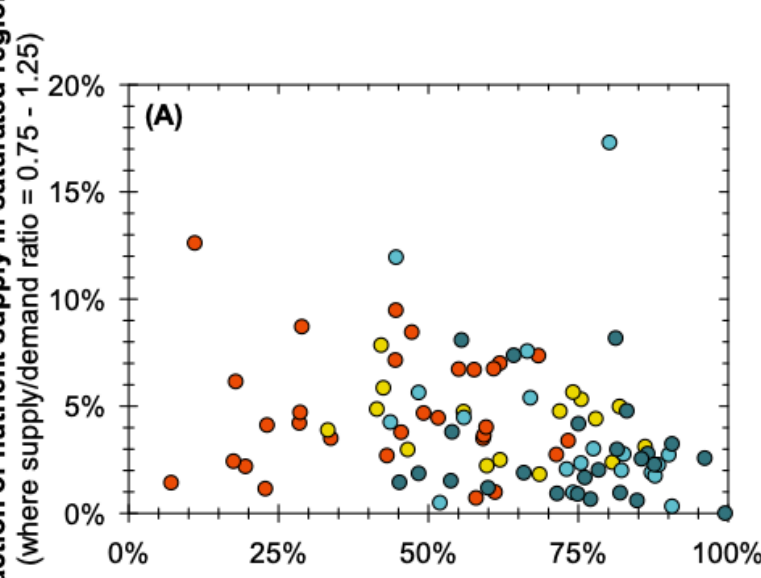

Fraction of nutrient supply below saturated region (where supply/demand ratio < 0.75)

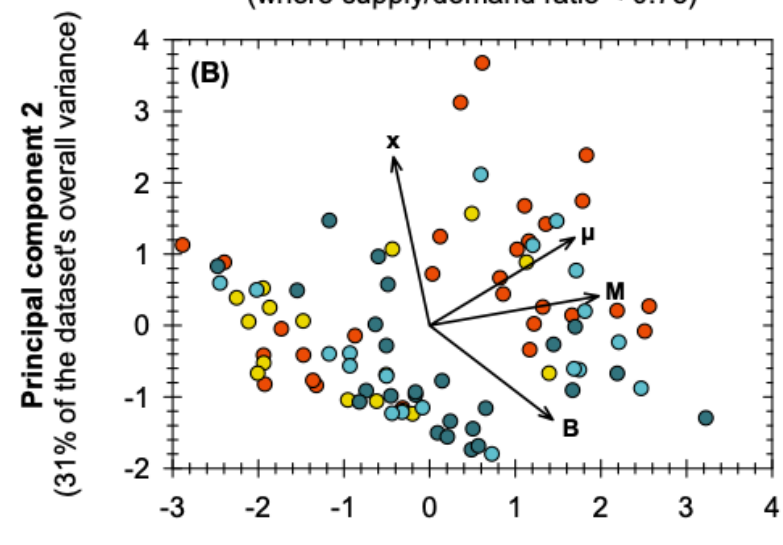

Principal component 1

(representing $54 \%$ of the dataset's overall variance)

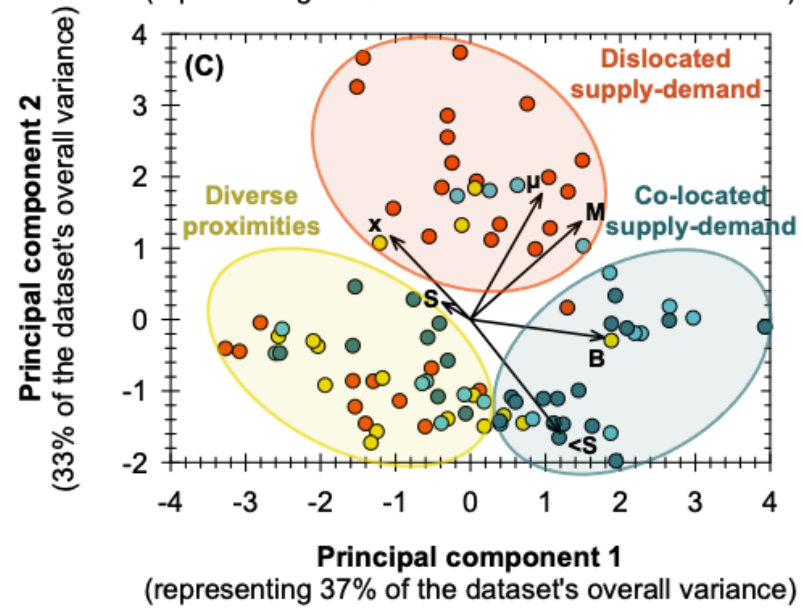

- HDI $1 \circ \mathrm{HDI} 2 \circ \mathrm{HDI} 3 \circ \mathrm{HDI} 4$

Figure S5. Potassium principal component analysis (PCA) using HDI grouping. PCA using $\mathrm{K}$ datasets and $\mathrm{HDI}$ grouping to identify clusters using $(A)$ data-derived parameters, $<S$ and $S$, (B) logistic function fitting parameters (after log transforming $B$ and $\mu$ parameters), and $(C)$ a combination of both. Classification of countries into each typology (co-located, dislocated, and diverse proximities) for potassium was based on Figure (C). Puerto Rico and Somalia were excluded because they did not have a HDI measurement; thus, only 90 countries are shown. 
Table S7. Nitrogen, phosphorus, and potassium PCA results showing clusters and country characteristics. Clusters are defined as co-located supply-demand ("Co-located"), dislocated supply-demand ("Dislocated"), and diverse proximities ("Diverse").

(Table $S 7$ is included as an excel spreadsheet:

Echevarria_et_al_-_Table_S7_NPK_PCA_results_92_countries.xlsx) 


\section{Section S3. Nutrient Saturation Profile Plots of All Countries}

A separate PDF file is available with nutrient saturation profiles for nitrogen, phosphorus, and potassium for 105 countries. Although 107 countries were evaluated for fitting parameters (Tables S4, S5, and S6), Seychelles (which included only two grid cells) and South Sudan (due to limited data which resulted in $99.9 \%$ of nutrient supply estimated to be in demand-limited/nutrient island grid cells) were not plotted.

Section 3 is provided as a separate file, in part, to help avoid the accidental printing of 105 color pages (i.e., Section S3) by readers who choose to print out the Supporting Information.

(Section S3 is included as an independent PDF:

Echevarria_et_al_-_Section_S3_Nutrient_Saturation_Profile_Plots_105_Countries.pdf)

\section{References}

(1) Wold, S.; Esbensen, K.; Geladi, P. Principal Component Analysis. Chemom. Intell. Lab. Syst. 1987, 2 (1), 37-52. https://doi.org/10.1016/0169-7439(87)80084-9.

(2) Abdi, H.; Williams, L. J. Principal Component Analysis. WIREs Comput. Stat. 2010, 2 (4), 433-459. https://doi.org/10.1002/wics.101. 